\title{
Physics and Performance Evaluation Group
}

\author{
Andrea Donini , Patrick Huber ${ }^{\dagger}$, Silvia Pascoli , \\ Walter Winter ${ }^{\ddagger}$ and Osamu Yasuda ${ }^{\S}$ \\ Instituto Física Teórica UAM/CSIC, Cantoblanco, E-28049 Madrid, Spain \\ ${ }^{\dagger}$ Physics Department, Theory Division, CERN, CH-1211 Geneva 23, Switzerland \\ IPPP, Department of Physics, Durham University, Durham DH1 3LE, United Kingdom \\ $¥$ Institut für theoretische Physik und Astrophysik, Universität Würzburg, D-97074 Würzburg, Germany \\ ${ }^{\S}$ Department of Physics, Tokyo Metropolitan University, Minami-Osawa, Hachioji, Tokyo 192-0397, Japan
}

\begin{abstract}
We summarize the objectives and results of the "international scoping study of a future neutrino factory and superbeam facility" (ISS) physics working group. Furthermore, we discuss how the ISS study should develop into a neutrino factory design study (IDS-NF) from the point of view of physics and performance evaluation.
\end{abstract}

Keywords: Neutrino Oscillations, Neutrino Factory

Preprint: IPPP/07/95, DCPT/07/190

PACS: $14.60 . \mathrm{Pq}$

\section{INTRODUCTION}

It is the main objective of any future neutrino factory, beta beam, or superbeam neutrino oscillation facility to provide information on $\sin ^{2} 2 \theta_{13}$, the neutrino mass hierarchy, and leptonic $\mathrm{CP}$ violation. These and other observables, such as deviations from maximal atmospheric neutrino mixing, turn out to be indications in favor of theories of lepton masses and mixings (see, e.g., Refs. [1, 2]). In addition, a detection of leptonic $\mathrm{CP}$ violation might be a good motivation to suspect the origin of the baryon asymmetry of the universe in the lepton sector. Any information from any such future neutrino oscillation facility can furthermore provide hints for new physics.

\section{ISS SUMMARY}

The physics case for a future accelerator-based neutrino oscillation facility was therefore made in Ref. [3] within the framework of the "International scoping study of a future neutrino factory and superbeam facility" (ISS) (for earlier studies, see Refs. $[4,5])$. The physics working group of this year-long study focused on "establishing the physics case for the various facilities and in finding the optimum parameters of the accelerator facility and detector systems from the physics point of view". It consisted of four major subgroups. The theory subgroup

\footnotetext{
E-Mail: andrea.donini@uam.es

$\dagger$ E-Mail: pahuberevt.edu

E-Mail: silvia.pascoliedurham.ac.uk

$¥$ Speaker, E-Mail: winter@physik.uni-wuerzburg. de

$\S$ E-mail: yasuda@phys.metro-u.ac.jp
}

identified the big questions which are essential for the physics case, such as questions related to the origin of neutrino mass, the role of neutrinos in the early universe, information from neutrinos about unification of forces and matter, etc.. The phenomenology subgroup discussed searches for clues of new physics in neutrino facilities alone, and in combination with other experiments. The experimental subgroup performed a detailed comparison of the performance of the various facilities. The muon physics subgroup reviewed muon physics that can be studied with the high intensity muon beam available at a neutrino factory.

The key objective of the ISS report [3] is to present the first detailed comparison of the performance of various facilities. The following represents the executive summary results with respect to the neutrino factory. Using realistic specifications, the likely performance is estimated, optimum combinations of facilities, baselines, and neutrino energies, are tried to be found, and some staging scenarios are attempted to be identified. Although the neutrino factory can achieve very large data samples with small backgrounds, it operates at energies considerably higher than the first oscillation peak $\left(E_{\max }=\mathrm{GeV}=L=564 \mathrm{~km}\right)$. Because of this, at intermediate values of $\theta_{13}\left(10^{3} \cdot \sin ^{2} 2 \theta_{13} \cdot 10^{2}\right)$ the neutrino factory with only one golden-channel $\left(v_{e} ! v_{\mu}\right.$ and $\bar{v}_{e} ! \bar{v}_{\mu}$ ) detector (at, say, $4000 \mathrm{~km}$ ) can not resolve all parameter degeneracies and the precision of the measurement of a particular parameter is reduced by correlations among the parameters. These problems can be resolved in one of three ways:

1. Placing a second detector at a different baseline (i.e. varying the ratio $L=E$ ); 
2. Adding a detector sensitive to the silver channel $\left(v_{e} ! v_{\tau}\right)$; or

3. Using an improved detector with lower neutrinoenergy threshold and better energy resolution.

Possible configurations for each alternative, alone and in combination, were investigated to find an optimum performance of the neutrino factory. It was shown that a considerable reduction of parent muon-energy down to $25 \mathrm{GeV}$ is feasible without a significant loss of oscillation-physics output, provided a detector performance improved with respect to the one assumed in earlier studies can be achieved.

As one of the results, we show in Figure 1 the CP violation and mass hierarchy discovery reaches of various proposed facilities. The figure shows the fraction of all possible values of $\delta$ for which $\mathrm{CP}$ violation (or the mass hierarchy) can be discovered as a function of the simulated $\sin ^{2} 2 \theta_{13}$. Three representative superbeam configurations were considered: the SPL, a superbeam directed from CERN to the Modane laboratory; T2HK, an upgrade of the J-PARC neutrino beam illuminating a detector close to Kamioka, and the WBB, a wide-band, on-axis beam from BNL or FNAL to a deep underground laboratory in the US. Each superbeam was assumed to illuminate a megaton-class water Cherenkov detector. The beta beam options considered were the CERN baseline scheme, in which helium and neon ions are stored with a relativistic $\gamma$ of 100, and an optimized beta beam, for which $\gamma=350$ ("BB" in Figure 1). Two neutrino factory options were considered: a conservative option with a single $50 \mathrm{kton}$ detector sited at a baseline $2000 \mathrm{~km}$ $4000 \mathrm{~km}$ from a $50 \mathrm{GeV}$ Neutrino Factory; and the optimized Neutrino Factory (see the full report) with two detectors, one at a baseline $2000 \mathrm{~km}-4000 \mathrm{~km}$ and the second at the magic baseline $(7500 \mathrm{~km})$. The result of the comparisons may be summarized as follows: for the options considered, the neutrino factory has the best discovery reach for $\sin ^{2} 2 \theta_{13}$ followed by the beta beam and the superbeam, while the $\sin ^{2} 2 \theta_{13}$ reach for resolving the sign of the atmospheric mass difference is mainly controlled by the length of the baseline. For large values of $\theta_{13}\left(\sin ^{2} 2 \theta_{13} \& 10^{2}\right)$, the three classes of facility have comparable sensitivity for the discovery of $\mathrm{CP}$ violation; the best precision on individual parameters being achieved at the Neutrino Factory using optimized detectors. The reduction of systematic uncertainties is the key issue at large $\theta_{13}$; by reducing systematic uncertainties, the superbeam may be favorably compared with the conservative neutrino factory. For intermediate values of $\theta_{13}\left(10^{3} \cdot \sin ^{2} 2 \theta_{13} \cdot 10^{2}\right)$, the superbeams are outperformed by the beta beam and the Neutrino Factory and the best CP coverage is achieved by the beta beam. For small values of $\theta_{13}\left(\sin ^{2} 2 \theta_{13} \cdot 10^{3}\right)$, the neutrino factory outperforms the other options. Note, the compar- isons are made using three performance indicators only $\left(\sin ^{2} 2 \theta_{13}\right.$, the sign of mass hierarchy and the CP violating phase $\delta$ ). If other physics topics, such as the search for $e ; \mu ; \tau$ flavor anomalies, were to be emphasized, the relative performance may be different. From the physics point of view, many of the different physics performance regions still overlap because of yet unclear systematics, luminosities, etc., which make a relative comparison of different approaches very challenging. Therefore, from any future design study, reliable predictions for these parameters as well as cost estimates will be required.

\section{IDS-NF PERSPECTIVES}

As for a neutrino factory, an international design study (IDS-NF) will continue the activities of the ISS. This follow-up study aims to present a design report, schedule, cost estimate, risk assessment for a neutrino factory. A part of this study will be the "physics and performance evaluation group" (PPEG). At least in the first phase, the focus will be on the performance of the various neutrino facilities. At a later stage, the focus will be shifted towards the physics case for a neutrino factory, and requirements for muon physics and non-standard physics will be included. The tasks of the PPEG include the coordination of the physics performance study, the maintenance of a web site, the interface with accelerator and detector working groups, possibly the organization of workshops, and providing the necessary reports.

After the ISS study, there are still many open questions to be addressed. For example, the baseline design has to be fixed, in particular, in discussion with the detector working group. Furthermore, new ideas have to be evaluated. For example, for relatively large $\sin ^{2} 2 \theta_{13}$, the possibility to have a low-energy neutrino factory has been drawing some attention $[8,9]$, possibly in combination with a superbeam [10]. Furthermore, the neutrino factory physics potential has to be compared to the one of other experiment classes, such as beta beams. The requirements for non-standard physics measurements, such as the silver channel [11], have to be defined. Finally, the requirements for muon physics and how these interact with the oscillation program ought to be investigated.

The main purpose of the PPEG is to evaluate the physics performance of a given experimental setup (neutrino factory or other, existing or planned) in a transparent, consistent and documented fashion. Hereby transparent means clearly stating the definition of the performance indicator, the approximations used, the chosen input parameters, the luminosity, the confidence level, and the treatment of systematics. In addition, consistent means that the assumptions should be on equal footing, and documented means that the relevant information will be archived and will be accessible, possibly on a web 

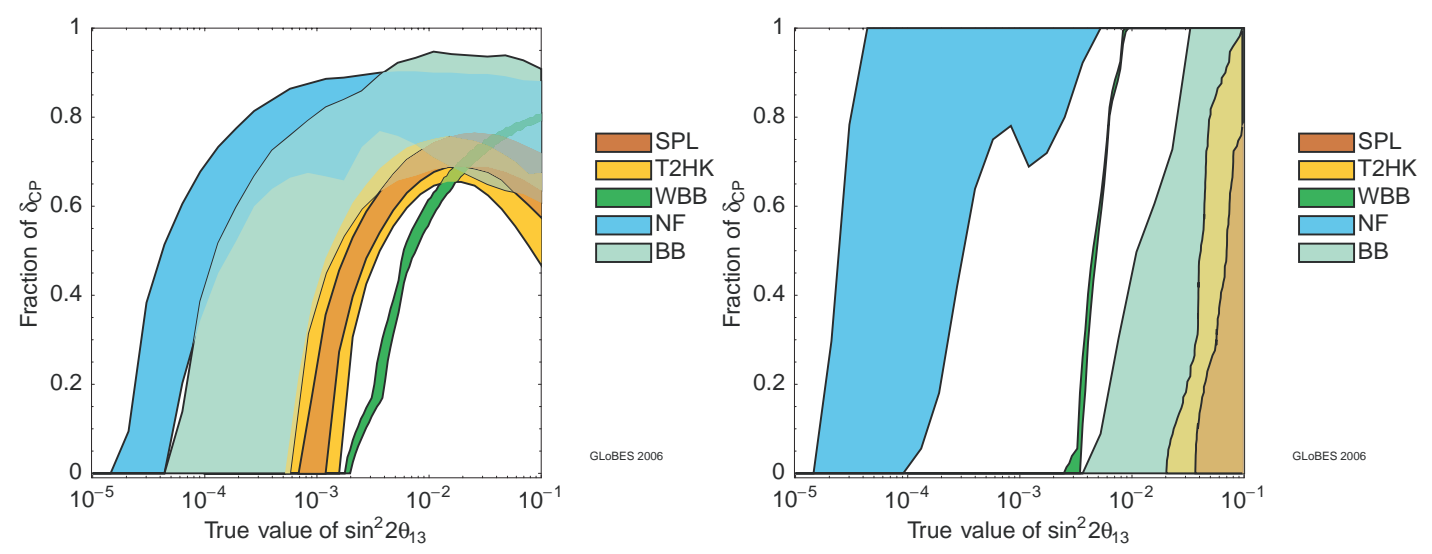

FIGURE 1. The CP violation (left) and mass hierarchy (right) discovery reaches of various proposed facilities. The figure shows the fraction of all possible values of $\delta$ for which CP violation or the mass hierarchy can be discovered as a function of the simulated $\sin ^{2} 2 \theta_{13}$. The right-hand edges of the bands correspond to conservative setups while the left-hand edges correspond to optimized setups. Figure from Ref. [3] computed using the GLoBES software [6, 7].

site. A major ingredient for this task is the definition of parameters. For the neutrino factory setups, all the parameters will be defined in strict collaboration with the detector and accelerator working groups of the IDS. This requires a defined and efficient communication between the various working groups. There might be different types of setups:

1. Baseline setup: stable, agreed design

2. Conservative modification of $1 .:$ for example, a different baseline

3. Speculative ideas

As for the beta beam setups, we will possibly follow the same procedure as for neutrino factory, working in collaboration with the beta beam groups. For the superbeam setups, the use of existing literature and input of wellestablished and recognized experts will be mandatory.

As far as the PPEG key ingredients are concerned, the main purpose will be performance evaluation following the method used in the ISS report. This means the use of GLoBES software [6, 7]. Performance plots should be updated twice a year and presented at NuFact. In Europe, there will be a close connection to the FP07 proposal, which is a funding proposal for a high-energy neutrino oscillation facility design study (in particular, between the IDS-NF PPEG and Euro $v$ design study Work Package 6: "Physics reach and comparison"). The PPEG has already set up a web site [12]. It will serve to illustrate the mandate, structure and activities of the PPEG. It will host the results of the performance evaluations. It will provide links to the documentation relative to the performance evaluation. And it will inform about the activities with announcements and with links to the workshops, the reports and any other relevant activity.

\section{SUMMARY}

In summary, the mandate of the PPEG is the continuation of the ISS activities, focusing on physics performance evaluation in a transparent, consistent, and documented. It is an international, inclusive effort, where everyone is invited to contribute.

Acknowledgments Some of us acknowledge the support of CARE, contract number RII3-CT-2003-506395. We would also like to thank the organizers of NuFact 07 for the nice meeting.

1. S. Antusch, P. Huber, J. Kersten, T. Schwetz, and W. Winter, Phys. Rev. D70, 097302 (2004), hep-ph/0404268.

2. C. H. Albright, and M.-C. Chen, Phys. Rev. D74, 113006 (2006), hep-ph/0608137.

3. The ISS Physics Working Group (2007), arXiv: 0710.4947 [hep-ph].

4. M. Apollonio, et al. (2002), hep-ph/0210192.

5. C. Albright, et al. (2004), physics / 0411123.

6. P. Huber, M. Lindner, and W. Winter, Comput. Phys. Commun. 167, 195 (2005), http: //www.mpi-hd.mpg.de/lin/globes/, hep-ph/0407333.

7. P. Huber, J. Kopp, M. Lindner, M. Rolinec, and W. Winter, Comput. Phys. Commun. 177, 432-438 (2007), hep-ph/0701187.

8. S. Geer, O. Mena, and S. Pascoli, Phys. Rev. D75, 093001 (2007), hep-ph/0701258.

9. A. D. Bross, M. Ellis, S. Geer, O. Mena, and S. Pascoli (2007), arXiv:0709.3889 [hep-ph].

10. P. Huber, and W. Winter, Phys. Lett. B655, 251-256 (2007), arXiv:0706.2862 [hep-ph].

11. A. Donini, D. Meloni, and P. Migliozzi, Nucl. Phys. B646, 321-349 (2002), hep-ph / 0206034.

12. IDS-NF PPEG, website (2007), http://www.ippp.dur.ac.uk/ids/. 